\title{
Familiarization Effects on Word Intelligibility in Dysarthric Speech
}

\author{
Heejin Kim ${ }^{a}$ Suzanne Nanney ${ }^{b}$ \\ ${ }^{a}$ Beckman Institute for Advanced Science and Technology, and ${ }^{b}$ Department of Speech and Hearing Science, \\ University of Illinois at Urbana-Champaign, Urbana-Champaign, III., USA
}

\section{Key Words}

Familiarization effects · Dysarthria - Speech intelligibility of dysarthric speech and support the efficacy of familiarization paradigms as an intervention technique in the management of dysarthria.

(c) 2015 S. Karger AG, Basel

\begin{abstract}
Background/Aims: This study investigated the effects of familiarization on naïve listeners' ability to perceive dysarthric speech produced by speakers with cerebral palsy and evaluated the degree of intelligibility improvement, both in the short and long term, as a function of (1) familiarization paradigms and (2) the number of familiarization phases. Methods: A total of 120 listeners (30 listeners/speaker) were recruited to complete word transcription tasks over a 6-week period. The listeners were assigned to one of the following familiarization paradigms: passive familiarization with audio signal only, active familiarization with both audio and orthography, and no explicit familiarization. Intelligibility scores were measured as the percentage of words correctly transcribed. Results: The active familiarization paradigm that provided listeners with both audio and orthography resulted in higher intelligibility scores compared to the passive familiarization and no explicit familiarization conditions. The degree of intelligibility improvement as a function of passive familiarization varied depending on the speaker. Last, the advantage of active familiarization was also found as a longterm effect. Conclusion: Our findings provide evidence for the benefits of familiarization in enhancing the intelligibility
\end{abstract}

\section{Introduction}

Individuals with dysarthria are affected by reduced speech intelligibility in their daily communication. Researchers have made substantial efforts to discover which aspects of the speech signal can be modified to lead to a significant gain in intelligibility. For example, numerous studies have investigated the effectiveness of speaking style manipulations in the remediation of dysarthric speech. Findings generally support that speaking style manipulations such as producing loud or clear speech enhance the acoustic distinctiveness of segments and, consequently, overall speech intelligibility [1-8]. This line of research targets speaker-related factors in pursuing intelligibility improvement in dysarthric speech, i.e., the main question in this approach is 'what can a speaker do to improve his or her speech intelligibility?'.

Because communication entails mutual speaker-listener interactions [9], researchers agree that to bring the intelligibility enhancement to its full potential, it is necessary to consider listener-related factors in addition to

\section{KARGER}

E-Mail karger@karger.com www.karger.com/fpl 
speaker-related factors [10-12]. Borrie et al. [13] further noted that research on familiarity, i.e., the listener's previous contact with or exposure to an acoustic signal [14], might provide a critical key to optimizing communicative success for speakers with dysarthria. Although findings generally suggest that experience with dysarthric speech improved a listener's ability to understand the speech, the gain size in intelligibility varies across studies [2, 13-19]. In addition, no significant effect of familiarization was found by Garcia and Cannito [20] and Yorkston and Beukelman [21]. Different degrees of success in familiarization-induced intelligibility improvement appear to be related to different methods across previous studies. For example, studies differed in terms of familiarization conditions. Hustad and Cahill [16] and Garcia and Cannito [20] employed a passive familiarization condition in which listeners were presented with an audio signal of dysarthric speech only, while other studies used an active familiarization condition in which listeners were familiarized both with the audio signal and a written transcript. The studies of Yorkston and Beukelman [21] and Borrie et al. [13] are the only ones that compared passive with active familiarization conditions, and their findings are conflicting. Yorkston and Beukelman [21] reported no group difference, while Borrie et al. [13] found the most robust intelligibility gains in the active familiarization condition. Studies also varied in terms of listener criteria: most used naïve listeners, while Yorkston and Beukelman [21] employed experienced listeners.

In pursuing research on the familiarization effects in dysarthric speech, questions remain concerning the following issues. First, previous work has suggested that different paradigms of familiarization may induce different degrees of intelligibility gains, but few studies systematically compared the effects of various familiarization conditions, leaving an optimal familiarization paradigm yet to be discovered. Second, previous findings have suggested a possible interaction between the severity of dysarthria and the magnitude of familiarization effects. For example, Hustad and Cahill [16] examined changes in intelligibility across four familiarization phases, whereas most studies employed only one familiarization phase. An interesting finding was that the point at which intelligibility gains became significant varied depending on the dysarthria severity level of the speaker. Specifically, the benefit of the listeners' familiarization became significant at a later trial for speakers with severe dysarthria compared to speakers with mild dysarthria. This finding suggests that the lack of intelligibility improvement found by Garcia and Cannito [20] might be attributable to the fact that the speaker in their

Familiarization Effects on Dysarthric Speech Perception study had severe dysarthria and listeners had only one familiarization phase. Since most studies employed an across-speaker familiarization method or examined only one level of dysarthria severity, it is still unclear whether a certain familiarization condition induces significant intelligibility gains regardless of the dysarthria severity of the speaker or whether there is a speaker-specific or severityspecific method. In addition, long-term retention of familiarization has been reported for second-language learners $[22,23]$, but no such work has focused on dysarthria [13].

The purpose of this study was to investigate the effects of familiarization - i.e., a brief prior exposure to a dysarthric speaker's speech - on naïve listeners' ability to transcribe single words produced by the dysarthric speaker. This study examined the speaker-specific familiarization in order to investigate possible interactions among familiarization paradigms, the number of familiarization phases, and speakers and/or dysarthria severity levels. To assess the efficacy of the different familiarization paradigms, this study compared the following three conditions: passive familiarization (i.e., only audio signal provided), active familiarization (i.e., both audio and a written transcript provided), and no explicit familiarization (only repetition of transcription tasks as in Hustad and Cahill [16]). In addition, familiarization effects were examined across four familiarization phases, adopting the methods of Hustad and Cahill [16]. Lastly, concerning the longterm retention of familiarization, this study examined whether the improvement in intelligibility due to familiarization is retained after 1 month.

The following research questions were addressed: (1) does the gain size in intelligibility vary depending on the familiarization paradigm?, (2) is there a familiarization paradigm that is effective for all speakers?, and (3) are familiarization effects retained in the long term?

\section{Materials and Methods}

This study was conducted as part of a larger research project, in which all listeners participated in both word and consonant transcription tasks. Since the focus of this study is on word transcription, only materials and methods related to word transcription tasks are described below.

\section{Stimuli}

Recordings of 4 speakers were extracted from the Universal Access (UA) corpus, which was originally developed as a resource for the automatic speech recognition technology development for speakers with dysarthria [24]. The UA database consists of dysarthric speech samples produced by native speakers of American English with cerebral palsy. To create the database, speakers were asked to read an isolated word displayed on a computer screen. 
Each speaker produced a total of 765 isolated words that included 3 repetitions of digits, computer commands (e.g., copy, cut), radio alphabet (e.g., tango, zulu), and common words (e.g., people, water), and 1 repetition of 300 uncommon words (e.g., giggled, zipper). The intelligibility levels of the speakers were estimated based on word transcription tasks by 5 native listeners/speaker, and the speakers were then classified into one of the following operationally defined intelligibility categories: very low (0-25\%), low (26$50 \%)$, mid (51-75\%), and high (76-100\%). For the current study, 4 speakers whose intelligibility was in the low or mid range were chosen considering the possibility of ceiling and floor effects, i.e., the familiarization effects might be minimal for the speakers at the extremes of intelligibility. All 4 speakers were from North or Central Illinois (USA) and were diagnosed with spastic dysarthria. Their age, gender, and intelligibility were as follows: Speaker $1=$ 58 years, male, 28\%; Speaker $2=30$ years, female, 29\%; Speaker $3=18$ years, male, $39 \%$, and Speaker $4=21$ years, male, $59 \%$.

The stimuli for this study were chosen based on the following criteria: (a) words must be mono- or bisyllabic; (b) words must have lexical stress on the first syllable, and (c) words must contain 1 of 16 consonant phonemes (/p, b, t, d, k, g, m, n, s, z, J, h, l, r, w, $\mathrm{j} /$ ) in the word initial position. The purpose of criterion (a) was to control a possible effect of linguistic complexity on the familiarization effects. Criteria (b) and (c) were chosen to control the distribution of consonants since our extended research will include analyses on consonant transcriptions. Four words were chosen to represent each of the 16 consonants, yielding a total of 64 distinct words. For familiarization phases, 64 stimuli were constructed using two acoustically distinct productions of 32 words. The stimuli for the word transcription task were 64 words consisting of 32 words that were different productions of the words introduced during the familiarization phase and 32 novel words. The peak amplitude of the stimuli was normalized to $69 \mathrm{~dB}$.

\section{Listeners and Experimental Groups}

Listeners were recruited using the following criteria: (a) native speakers of American English; (b) between 18 and 40 years old; (c) no identified language, learning, or cognitive disability per self-report; (d) no more than incidental experience with persons with language disorders; (e) grew up in the North Midland dialect region, and (f) passed a hearing screening at $20 \mathrm{~dB}$ for $250,500,1,000,2,000$, $4,000,6,000$, and $8,000 \mathrm{~Hz}$. Thirty listeners were randomly assigned to each speaker, with the condition of 10 listeners in each of the 3 experimental groups, i.e., Group A (audio only) for passive familiarization, Group B (both audio and orthography) for active familiarization, and Group C (controls) for no explicit familiarization.

\section{Procedure}

The listeners were seated directly in front of a laptop computer screen in a sound-treated room. The stimuli were presented via loudspeaker approximately 2 feet away from the listeners. The peak output level of the stimulus material was approximately 67 $\mathrm{dB}$ at the location where the listeners were seated. A custom-built experimental interface was used to play stimuli and collect the listeners' transcriptions. The listeners participated in 3 experimental sessions over a 6 -week period. Before starting each day of the experiment, the listeners completed a practice round to get acquainted with the experimental interface. They were told that all words that they would hear would be real words in American English that were produced by an individual with a speech disorder.
During Session 1 (pre-familiarization session), all listeners completed one round of word transcription tasks. For these tasks, the listeners were asked to identify the word they heard by typing it into the computer. The stimuli were presented one at a time and only once. The listeners were instructed to give their best guess even if they were unsure of the intended word. The data from Session 1 served to establish baseline intelligibility. One week later, the listeners returned to complete Session 2, which consisted of four rounds of word transcription tasks. Before each round of transcription tasks, the listeners in Group A underwent a familiarization phase in which they heard a series of 64 words produced by their assigned speaker; this was the passive familiarization condition. The listeners in Group B heard words while reading a corresponding orthographic representation of the target word on the computer screen; this was the active familiarization condition. The listeners in Group $\mathrm{C}$ received no familiarization phase but completed four rounds of transcription tasks; this was the control condition. Approximately 1 month after Session 2, the listeners returned to complete Session 3 (long-term, delayed test), during which they completed the same round of word transcription tasks as in Session 1.

\section{Analysis}

The transcribed words were scored as either correct or incorrect using a customized computer program, which calculated the percentage of correct responses per listener. Homonyms of target words were scored as correct. The accuracy of the computer scoring was manually checked by the first author for every transcript. Whenever the author found that the computer program had scored misspelled words or typographical errors as incorrect, they were changed to correct; corrections were made for less than $1 \%$ of the data.

A two-way mixed ANOVA analysis was conducted separately for each speaker. Instead of merging the data across the speakers, within-speaker analyses were chosen to address the question whether different familiarization paradigms induce significant intelligibility improvement for only certain speakers or for all speakers. The dependent measure was the intelligibility scores (i.e., the percentage of correct words). The between-group-independent factor was 'Group', which represented the three familiarization paradigms. The repeated measure was 'Session', for which categories were Session 1, Session 2-first round, Session 2-second round, Session 2-third round, Session 2-fourth round, and Session 3 (henceforth referred to as 'Session 1, Session 2-1, Session 2-2, Session 2-3, Session 2-4, and Session 3'). Prior to the main analyses, a one-way ANOVA was conducted on Session 1 scores for each speaker individually to verify that the listeners' baseline performance before familiarization was uniform across the experimental groups. The ANOVA results confirmed that Session 1 scores were not significantly different among the different groups for a given speaker.

\section{Results}

Figure 1 presents the mean intelligibility scores of Session 1 (i.e., pre-familiarization scores), the highest mean intelligibility scores among four rounds in Session 2, and the mean intelligibility scores of Session 3 (i.e., 1-month delayed test) for the three experimental groups. Across all 


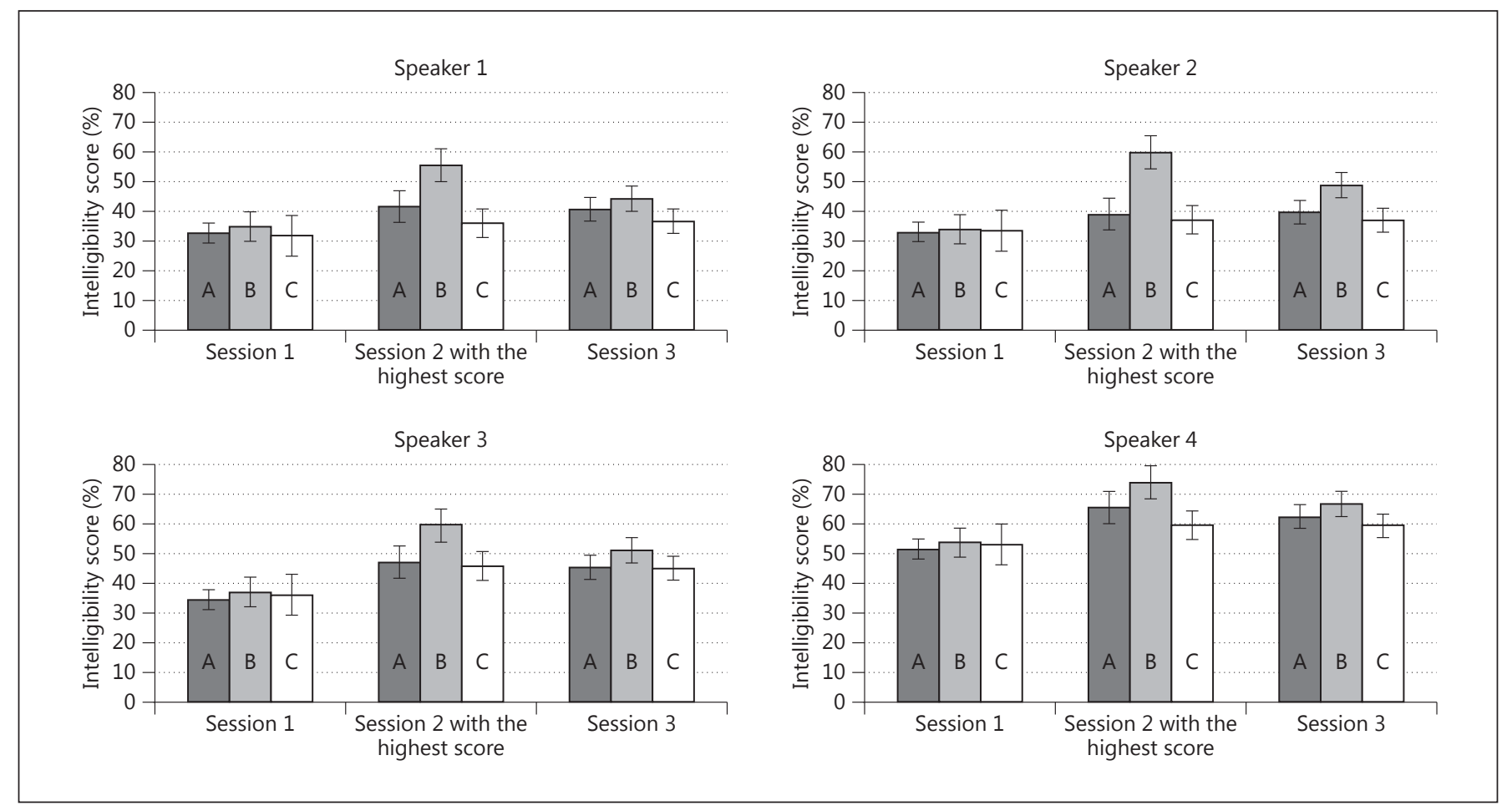

Fig. 1. Mean intelligibility scores ( \pm 1 SD) are listed against 3 sessions: Session 1 (pre-familiarization session), one of the Session 2 rounds with the highest score, and Session 3 (1-month delayed session). Experimental groups A (audio only), B (both audio and orthography), and $\mathrm{C}$ (control) are shown in dark gray, light gray, and white bars, respectively.

speakers, Session 2 scores were higher than Session 1 scores for all experimental groups, and Group B (active familiarization) showed higher Session 2 scores than Group A (passive familiarization) and Group C (controls). While Session 3 scores were comparable to or lower than Session 2 scores, they were higher than Session 1 scores for all experimental groups across all speakers. In addition, as in Session 2 scores, Group B showed higher Session 3 scores than the other two groups.

To illustrate the changes in the intelligibility scores over time, figure 2 shows the mean scores of each session for the three experimental groups. For all 4 speakers, listeners in Group B (active familiarization) exhibited higher scores than listeners in Group A (passive familiarization) and Group C (controls) in all of the Session 2 rounds as well as in Session 3. The ANOVA for Group $\times$ Session revealed that all effects, including the interaction, were significant at $\mathrm{p}<0.05$ for all speakers. Specifically, there was a significant main effect of Group on the percent scores for all speakers: for Speaker 1, F $(2,26)=24.06$; for Speaker 2, F (2,27) = 29.89; for Speaker 3, F $(2,27)=23.23$, and for Speaker 4, F $(2,27)=14.67$. Also, there was a sig- nificant main effect of Session for all speakers: for Speaker $1, \mathrm{~F}(5,130)=39.73$; for Speaker 2, F $(5,135)=48.92$; for Speaker 3, F $(5,135)=70.42$, and for Speaker 4, F(5, $135)=95.61$. In addition, the Group $\times$ Session interaction was significant for all speakers: for Speaker 1, F(10, 130 $)=9.38$; for Speaker 2, F $(10,135)=22.6$; for Speaker $3, F(10,135)=7.05$, and for Speaker 4, F $(10,135)=10.02$. The significant interaction indicates that the Session effect varied depending on Group: as shown in figure 2, Group B (active familiarization) exhibited a larger degree of increase over sessions compared to Group A (passive familiarization) and Group C (controls).

The results of subsequent post hoc analyses based on Tukey tests are summarized in table 1; speakers are listed in increasing order of intelligibility, from the most severe to the least severe. As shown in this table, significant differences were found among the experimental groups for all 4 speakers. A major finding across all speakers was that Group B (active familiarization) was distinct from Group C (controls) for all sessions. Speaker-specific patterns were also found especially in the relation of Group A (passive familiarization) to the other groups. For Speaker 2 

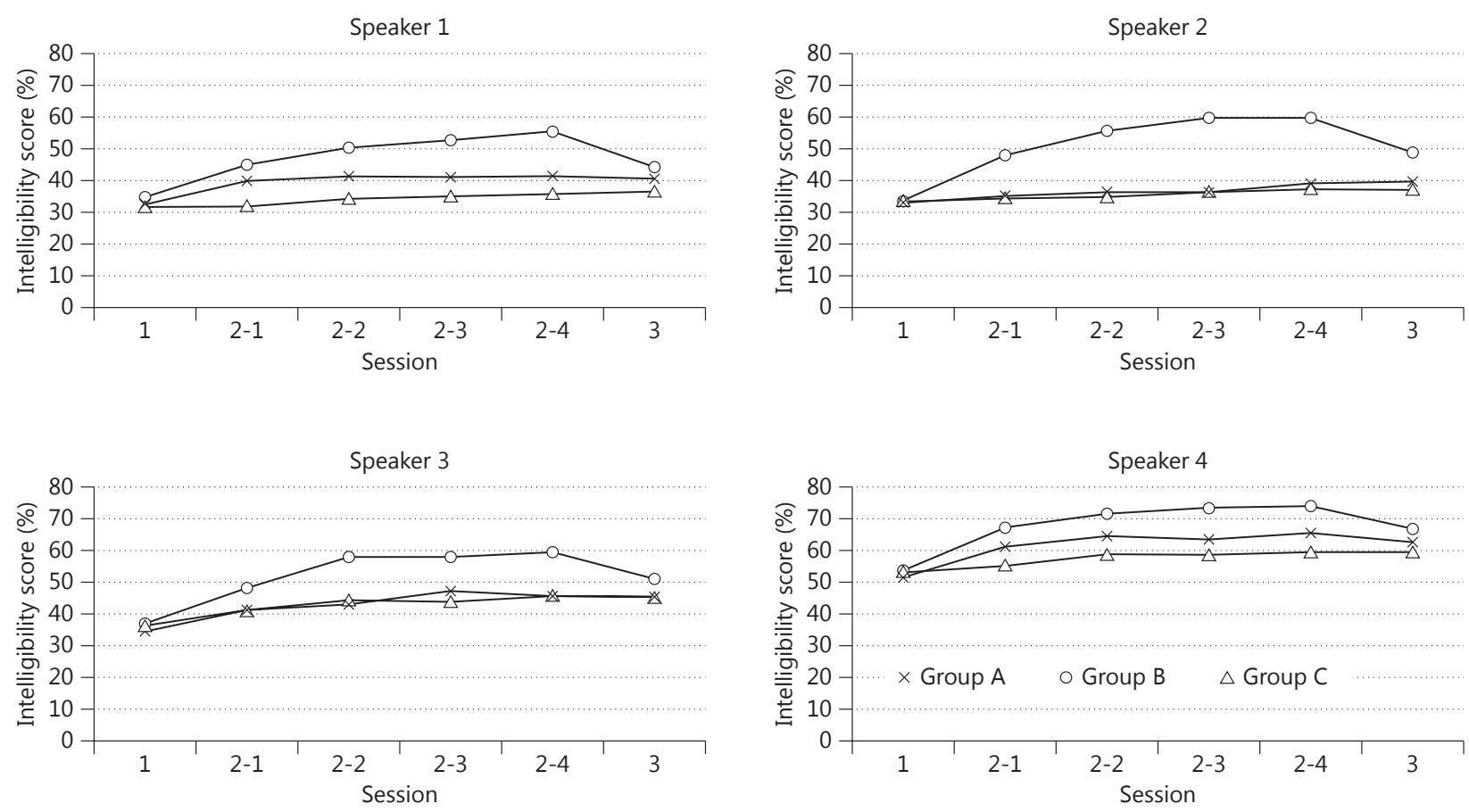

Fig. 2. Mean intelligibility scores plotted against each session for the three experimental groups.

Table 1. Results of the post hoc (Tukey) analysis of the experimental groups for intelligibility scores

\begin{tabular}{llllll}
\hline Speaker & Session 2-1 & Session 2-2 & Session 2-3 & Session 2-4 & Session 3 \\
\hline 1 & $\mathrm{~B}=\mathrm{A}>\mathrm{C}$ & $\mathrm{B}>\mathrm{A}>\mathrm{C}$ & $\mathrm{B}>\mathrm{A}>\mathrm{C}$ & $\mathrm{B}>\mathrm{A}=\mathrm{C}$ & $\mathrm{B}=\mathrm{A}>\mathrm{C}=\mathrm{A}$ \\
2 & $\mathrm{~B}>\mathrm{A}=\mathrm{C}$ & $\mathrm{B}>\mathrm{A}=\mathrm{C}$ & $\mathrm{B}>\mathrm{A}=\mathrm{C}$ & $\mathrm{B}>\mathrm{A}=\mathrm{C}$ & $\mathrm{B}>\mathrm{A}=\mathrm{C}$ \\
3 & $\mathrm{~B}>\mathrm{A}=\mathrm{C}$ & $\mathrm{B}>\mathrm{A}=\mathrm{C}$ & $\mathrm{B}>\mathrm{A}=\mathrm{C}$ & $\mathrm{B}>\mathrm{A}=\mathrm{C}$ & $\mathrm{B}>\mathrm{A}=\mathrm{C}$ \\
4 & $\mathrm{~B}>\mathrm{A}>\mathrm{C}$ & $\mathrm{B}>\mathrm{A}=\mathrm{C}$ & $\mathrm{B}>\mathrm{A}=\mathrm{C}$ & $\mathrm{B}>\mathrm{A}>\mathrm{C}$ & $\mathrm{B}=\mathrm{A}>\mathrm{C}=\mathrm{A}$ \\
\hline
\end{tabular}

$\mathrm{A}, \mathrm{B}$, and $\mathrm{C}$ refer to the experimental groups $\mathrm{A}$ (passive familiarization), B (active familiarization), and C (control), respectively. Results are shown separately for each speaker and for each session. Speakers are listed in increasing order of intelligibility, from the most severe to the least severe.

and Speaker 3, Group A was distinguished from Group B in all sessions, but not distinct from Group $\mathrm{C}$ in any of the sessions. On the other hand, for Speaker 1, Group A was distinguished from Group B in all sessions except for Sessions 2-1 and 3, and distinct from Group $C$ in Sessions 2-1 and 2-2. For Speaker 4, Group A was distinguished from Group B in all sessions except for Session 3, while it was distinct from Group C in Sessions 2-1 and 2-4.

\section{Discussion}

\section{Speaker-General Effects}

Regarding the first research question, i.e., 'does the gain size in intelligibility vary depending on familiarization paradigms?', our results showed that for all speakers, the listeners in the active familiarization group achieved higher scores than the listeners in the passive familiariza- 
tion and control groups. This finding suggests that active familiarization through both audio and a written transcript is generally more successful in enhancing the intelligibility of dysarthric speech compared to the passive familiarization and simple repetition of transcription tasks. In addition, a significant interaction between Group and Session for all speakers confirmed that the degree of intelligibility enhancement as a function of Session was not uniform across the different experimental groups, but rather the Session effect was greater for the active familiarization group than for the other groups. A notable pattern general to all speakers was that the active familiarization group outperformed the controls in all sessions, verifying the advantage of using an active familiarization method over the method of no explicit familiarization.

Speaker-general effects are also found in the analyses on the long-term effects. Specifically, Session 3 (1-month delayed test) scores were higher than Session 1 scores for all three experimental groups and all speakers, confirming that familiarization-induced intelligibility enhancement is retained 1 month after familiarization to some degree. Although the active familiarization group showed the largest decline between Session 2-4 and Session 3 scores, this group still demonstrated the highest Session 3 scores compared to the passive familiarization and control groups, verifying the advantage of an active familiarization paradigm for the long-term effect of familiarization. The speaker-general effects of active familiarization in both the short and long term suggest that the familiarization by both audio and written material facilitates more robust intelligibility benefits compared to passive and simple repetition paradigms. Furthermore, the difference between active and passive familiarization in the magnitude of the intelligibility gain underlines the importance of including a written transcript alongside the audio signal during familiarization. Our findings extend support for the claim by Borrie et al. [13] and Norris et al. [25] that the presence of written material - in the current study, specifically lexical information - enables listeners to use lexical knowledge to adjust the mapping between acoustic-phonetic information and the phonemic representation in the language.

\section{Speaker-Specific Effects}

Speaker-specific patterns were found especially when comparing the passive familiarization group with the controls. For Speaker 2 and Speaker 3, the passive familiarization group was not distinguished from the controls in any of the sessions, implying that passive familiarization was ineffective to the same degree as no explicit familiarization for these speakers. In stark contrast, for Speaker 1 and

Familiarization Effects on Dysarthric Speech Perception
Speaker 4, the passive familiarization group showed higher scores than the control group in some sessions, especially in Session 2-1 (i.e., after the very first familiarization phase), suggesting that using passive familiarization for these speakers yielded some degree of success. The speaker-specific effects of passive familiarization cannot be attributed to the speakers' severity of dysarthria because Speaker 1 and Speaker 2, who exhibited contrasting results, were similar in terms of their base intelligibility scores.

The passive familiarization and control groups were different in terms of the amount of exposure to the audio signal: the first had a total of 8 repetitions of listening (4 familiarization phases +4 transcription tasks), while the latter only had 4 repetitions of listening. Given that the passive familiarization group's Session 2-2 scores were still higher than the controls' Session 2-4 scores for both Speaker 1 and Speaker 4, however, the possibility of the amount of exposure to audio signals as the main reason for the passive familiarization group's advantage over the controls is ruled out. It is important to note that the passive familiarization and control groups received qualitatively different audio signals: the listeners in passive familiarization listened to more acoustic variations of the target words than the controls (i.e., 3 varieties as opposed to 1). This leads us then to postulate that when the listeners are exposed to a rich acoustic variation of words, their perception of the speech may improve. Further research is needed to investigate what factors determine the degree of success for different familiarization paradigms, e.g., are the characteristics of acoustic deviances in dysarthric speech the key determinant of the effectiveness of passive familiarization?

To conclude, the current study found evidence for the benefit of familiarization in enhancing word intelligibility in dysarthric speech. The largest benefit was associated with the active familiarization paradigm that provided the listeners with both audio and orthographic information. The advantage of active familiarization over passive familiarization was also found in the long-term effect, but there was a decline in intelligibility 1 month after active familiarization. Future studies are needed to find a method that can maximize the long-term effect of familiarization (e.g., an interactive feedback protocol in which the listeners are given explicit feedback such as 'correct' or 'incorrect' in response during familiarization). The absence of further gains in intelligibility for passive familiarization compared to the control group was found for some speakers. This finding calls for a future study that examines the relation between the intelligibility gain size and the nature of the acoustic deviations in dysarthric speech. 


\section{Acknowledgements}

This study was supported by NIDCD R03DC012888A. We thank the subjects for their participation and Dr. Torrey Loucks for making a sound booth facility available for our data collection.
We further thank the reviewers for helpful comments. Part of this work was presented at the ASHA Annual Convention, Chicago, Ill., USA, November 14-16, 2013, and at the Motor Speech Conference, Sarasota, Fla., USA, February 27-March 2, 2014.

\section{References}

1 Beukelman DR, Fager S, Ullman C, Hanson E, Logemann J: The impact of speech supplementation and clear speech on the intelligibility and speaking rate of people with traumatic brain injury. J Med Speech Lang Pathol 2002; $10: 237-242$

-2 D’Innocenzo J, Tjaden K, Greenman G: Intelligibility in dysarthria: effects of listener familiarity and speaking condition. Clin Linguist Phon 2006;20:659-675.

3 Hanson EK, Beukelman DR, Fager S, Ullman C: Listener attitudes toward speech supplementation strategies used by speakers with dysarthria. J Med Speech Lang Pathol 2004; 12:161-166.

4 Rutter B: Acoustic Properties of Repair Sequences in Dysarthric Conversational Speech: An Interactional Phonetic Study (dissertation). Lafayette, University of Louisiana at Lafayette, 2008

5 Rutter B: Repair sequences in dysarthric conversational speech: a study in interactional phonetics. Clin Linguist Phon 2009;23:887900.

-6 Tjaden K, Wilding GE: Rate and loudness manipulations in dysarthria: acoustic and perceptual findings. J Speech Lang Hear Res 2004; $47: 766-783$

7 Tjaden K, Wilding G: The impact of rate reduction and increased loudness on fundamental frequency characteristics in dysarthria. Folia Phoniatr Logop 2001;63:178-186.

8 Yorkston K, Hakel M, Beukelman D, Fager S: Evidence for effectiveness of treatment of loudness, rate, or prosody in dysarthria: a systematic review. J Med Speech Lang Pathol 2007;15:11-36.
9 Lindbloom B: On the communication process: speaker-listener interaction and the development of speech. Augment Altern Commun 1990;6:220-230.

10 Hustad KC: Contribution of two sources of listener knowledge to intelligibility of speakers with cerebral palsy. J Speech Lang Hear Res 2007;50:1228-1240.

11 Hustad KC, Jones T, Dailey S: Implementing speech supplementation strategies: effects on intelligibility and speech rate of individuals with chronic severe dysarthria. J Speech Lang Hear Res 2003;46:462-474.

12 Patel R, Schroeder B: Influence of familiarity on identifying prosodic vocalizations produced by children with severe dysarthria. Clin Linguist Phon 2007;21:833-848.

13 Borrie SA, McAuliffe MJ, Liss JM, Kirk C, O’Beirne GA, Anderson T: Familiarisation conditions and the mechanisms that underlie improved recognition of dysarthric speech. Lang Cogn Process 2012;27:1039-1055.

14 Spitzer SM, Liss JM, Caviness JN, Adler C: An exploration of familiarization effects in the perception of hypokinetic and ataxic dysarthric speech. J Med Speech Lang Pathol 2000; 8:285-293.

15 Borrie SA, McAuliffe MJ, Liss JM: Perceptual learning of dysarthric speech: a review of experimental studies. J Speech Lang Hear Res 2012;55:290-305.

16 Hustad KC, Cahill MA: Effects of presentation mode and repeated familiarization on intelligibility of dysarthric speech. Am J Speech Lang Pathol 2003;12:198-208.
17 Liss JM, Spitzer SM, Caviness JN, Adler C: The effects of familiarization on intelligibility and lexical segmentation in hypokinetic and ataxic dysarthria. J Acoust Soc Am 2002;11: $2-6$

18 Tjaden K, Liss JM: The role of listener familiarity in the perception of dysarthric speech. Clin Linguist Phon 1995;9:139-154.

19 Tjaden K, Liss JM: The influence of familiarity on judgments of treated speech. Am J Speech Lang Pathol 1995;4:39-48.

20 Garcia JM, Cannito MP: Influence of verbal and nonverbal contexts on the sentence intelligibility of a speaker with dysarthria. J Speech Hear Res 1996;39:750-760.

21 Yorkston KM, Beukelman DR: The influence of judge familiarization with the speaker on dysarthric speech intelligibility; in Berry W (ed): Clinical Dysarthria. Austin, Pro-Ed, 1983, pp 155-164.

22 Bradlow AR, Akahane-Yamada R, Pisoni DB, Tohkura Y: Training Japanese listeners to identify English /r/ and /l/: long-term retention of learning in perception and production. Percept Psychophys 1999;61:977-985.

23 Nishi K, Kewley-Port D: Training Japanese listeners to perceive American English vowels: influence of training sets. J Speech Lang Hear Res 2007;50:1496-1509.

24 Kim H, Hasegawa-Johnson M, Perlman A, Gunderson J, Huang T, Watkin K, Frame S: Dysarthric speech database for universal access research. Proc Inter Speech 2008;17411744.

25 Norris D, McQueen JM, Cutler A: Perceptual learning in speech. Cogn Psychol 2003;47: 204-238. 